\title{
EINIGE BEMERKUNGEN ZU DEN SYNTAKTISCH - LEXIKALISCHEN RELATIONEN IN DER ERZÄHLUNG „AUS DEM LEBEN EINES TAUGENICHTS"
}

Die literarischen Texte als fiktive Textsorte liefern im linguistischen Sinne ein reiches Material zur Erforschung von Relationen im Sinne der grammatischen und inhaltlichen Selbständigkeit der Sätze, der Art ihrer Verbindungen im Großkontext. In Richtung der Verfolgung der gegenseitigen Verflochtenheit von selbständigen Sätzen und ihrer untrennbaren Verbindung im Rahmen des Großkontextes gingen die Untersuchungen seit Jahren (Vgl. Boost 1949; Admoni 1982: 250ff.; Eroms 2000).

Es meldet sich die Frage, wie weit und ob sich die Aufgaben von Dichtung im Kontext der stilistischen Anforderungen, der Einheitlichkeit formulieren lassen. Bei Schendel, Riesel wird betont, dass die Wirklichkeit in dichterischer Fiktion im Unterschied zu allen Funktional- und Substilen mit Hilfe künstlerischer Bildkraft wieder gespiegelt wird. Eroms spricht von der unterschiedlichen, autokonzentrierten Sprachhaltung, die bewirkt, dass die Leserschaft über die gewählten sprachlichen Formen eine Leseaugabe bekomme, in diesem Sinne bleibe die Lektüre von Dichtung immer rätselhaft. Stilistisch gesehen lässt sich die Dominanz des Individuellen neben der Fiktionalität als Wesen und wichtiger Bestandteil von literarischen Texten feststellen (Riesel, Schendel 1970: 20; Eroms 2008: 130).

Am Beispiel der Novelle „Aus dem Leben eines Taugenichts“ von Joseph von Eichendorff lassen sich die für den Aufbau der repräsentativen Erscheinungen von Textkohärenz notwendigen Merkmale identifizieren, indem die Sätze im Text nicht nur als eine bloße Folge, sondern als eine sprachliche Einheit zu interpretieren wären (Maingueneau 2000: 151).

Eichendorff wird als letzter deutscher Romantiker, oft auch eigentümlichster Dichter der Schule gesehen. Wir haben im Text mit der für den Romantismus typischen Ausdrucksweise zu tun, in der die Wirklichkeit über gegenseitige Verflochtenheit der Erscheinungen und Beziehungen der Sätze, ihres größeren oder geringe- 
ren Umfangs herausgehoben wird. Auf die Struktur des Textes sind auch das thematische Moment, die Konzeption der Hauptfiguren und die soziale Wirklichkeit nicht ohne Einfluss geblieben.

Die im Jahre 1826 in Danzig entstandene Novelle ist eine Ich - Erzählung, in der der Held und Erzähler sich identifizieren lassen, gibt dem Leser die Möglichkeit, von ersten Sätzen eine konkrete Haltung einzunehmen. Er wird von einer Zahl von Formeln angesprochen, die den Taugenichts als eine Idylle mit märchenhaften Zügen präsentieren. Wir haben zugleich mit Märchen und Wirklichkeit zu tun. Eine der Hauptformeln des Handlungsaufbaus ist das Wandern, zwar ohne Kenntnis fremder Sprachen, ohne eigentliche Ziele und Absichten, ohne Geldvorstellung also nicht zu realistisch (Bormann 1988: 352). Aber die Wanderung wird auch mit der Bedeutung versehen: Sohn eines Müllers besitzt zwar eine einfache Natur, ist aber zu einem Leben bereit und fähig. Sein Weggehen von daheim und fluchtartiges Verlassen der Einnehmerstelle werden vom Dichter als Sichwehren des Taugenichts, in die Umwelt seiner Zeit eingeordnet zu werden, motiviert. Flucht im Sinne der Auflehnung gegen das Leben, das die harmonische Ausbildung der Persönlichkeit gewährleistet. In der Opposition gegenüber konkreten Erscheinungen der Zeit erkennen wir den Realismus der Novelle. Der Taugenichts versteht die Gefahr der selbstgewählten Isolierung.

Alles ist so fröhlich, um dich kümmert sich kein Mensch. Und so geht es mir überall und immer. Jeder hat sein Plätzchen auf der Erde ausgesteckt, hat seinen warmen Ofen, seine Tasse Kaffee, seine Frau, sein Glas Wein zu Abend und ist so recht zufrieden ... Mir ist's nirgend recht. Es ist, als wäre ich überall eben zu spät gekommen, als hätte die ganze Welt gar nicht auf mich gerechnet.

Über Struktur der Sätze bekommen wir ein kohärentes Wirklichkeitsbild. Die Präsuppositionen helfen das Gesagte näher zu präsentieren. Tilgung der Prädikate, syndetische Kombinationen, Zusammensetzungen ergänzen das dynamische Bild des Textes.

Die syntaktische Struktur der direkten Rede lässt eine gewisse Verifizierung der eigenen Weltansicht zu, die einfache Beschreibung des Erzählers führt den Leser in die konkreten und abstrakten Schattierungen der Weltauffassung. Hier wäre noch der stilistisch relevante Gebrauch der Tempora zu erwähnen, und zwar Präsens oder Perfekt haben sich zum Ausdruck der besprochenen Welt am besten geeignet.

Das Wandern im Sinne der Bereitschaft wird von dem Leser mit der Jugendwelt wahrgenommen. Die armen Prager Theologiestundenten, die sich ein bißchen Geld als Wandermusikanten verdienen, lassen aus dem dialogischen Verhältnis von Welt und Ich erkennen, dass die jungen Menschen um so mehr leben, je weniger sie in ihrem Planen befangen bleiben (Stocklein 1987: 58).

Der Waldhornist sah mich gross an. Das tut gar nichts, so fuhr er ... fort, ich möchte gar nicht so reisen: Pferde und Kaffee und frisch überzogene Betten, und Nachtmützen und Stiefelknecht vorausbestellt. Das ist just das Schönste, wenn wir so frühmorgens heraustreten, und die Zugvögel hoch über uns fortziehen, dass wir gar nicht wissen, welcher Schornstein heut für uns raucht, und 
gar nicht vorausehen, was uns bis zum Abend noch für ein besonderes Glück begegnen kann. Ja, sagte der andere, und wo wir hinkommen und unsere Instrumente herausziehen, wird alles fröhlich ... und die Fräuleins an der Tafel verdrehen sich fast die Hälse, um die Musikanten draussen zu sehen. Wahrhaftig, rief der Waldhornist mit leuchtenden Augen aus, lasst die andern nur ihre Kompendien repetieren, wir studieren unterdies in dem grossen Bilderbuche, das der liebe Gott uns draussen aufgeschlagen hat! Ja, glaub nur der Herr, aus uns werden gerade die rechten Kerls, die den Bauern dann was zu erzählen wissen und mit der Faust auf die Kanzel schlagen, dass den Knollfinken unten vor Erbauung und Zerknirschung das Herz im Leibe bersten möchte.

Die Art der Verbundenheit der Sätze lässt gleichzeitig die Einstellung des Autors zur Verflechtung der Erscheinungen der Wirklichkeit erkennen, in der Struktur der Sätze, ihrem Umfang, Verwendung der hypotaktischen und parataktischen Verbindungsmittel sehen wir die Komplexität der logischen Beziehungen im Satzbau. Während z.B. Personalpronomina und Demonstrativpronomina eine Wiederholung, eine gewisse Verallgemeinerung erzielen, fällt den Konjunktionen noch eine andere Funktion hinzu, und zwar einer Brücke, rückwärts und vorwärts gerichtet. Die Konjunktion und drückt eine Angliederung aus, kann aber auch das Blickfeld erweitern, wenn sie die erste Position im Satz einnimmt und eine gewisse Weltweite erzielt (Vgl. Silman 1974: 34ff.).

Bei der Lektüre des Textes ist vor allem die Vielfalt Eichendorffs Lieblingsworte, die häufig im Text vorkommen, auffallend. Die Invarianten lassen den Persönlichkeitsstil des Dichters erkennen, indem er gewisse Motive immer neu zu erörtern versucht hatte. In seinem Wortschatz lassen sich die zentralen Motive des romantischen dichterischen Sprachstils gut verfolgen. In der Gruppe der Substantive treten statistisch am häufigsten folgende Lexeme hervor: Morgen, Wald, Welt, Ferne, Mond, Abend, Berg, Wasser, Frühling, Mühle.

Sie wären als globale Handlungsfelder aufzufassen und helfen, einzelne voraussehbare kommunikative Situationen zu fixieren. Um diese Hauptwörter gruppieren sich dann die Zusammensetzungen oder attributive Ergänzungen, die als Beispiele für eine gewisse Invarianz bestimmter Wörter und Wendungen dienen könnten. Die Inwarianten gelten auch als Auswirkungen eines ausgebildeten Persönlichkeitsstils. Dabei wären die Häufigkeitsziffer der Anwendung von bestimmten Worten kein Zeichen von Ausdrucksarmut sondern vielmehr der Tatsache, dass der Dichter von gewissen Ideen gebannt, sie stets von neuem kreativ anwendet. Diesen Lieblingsworten fällt die Aufgabe hinzu, als Symbole der das Fühlen, Dichten und Denken bestimmenden romantischen Motive aufzutreten. Hier wären die Belege zu nennen, die als Beispiele für die erwähnte Invarianz dienen könnten.

\footnotetext{
Morgen - Morgenscheine - Morgenstrahlen - Morgenlicht - Morgenstunde - Morgenkühle - Morgenstunde - Morgensonne - Morgenlied - Morgenduft - Morgenstille - Morgenstreifen - Morgenrote Wald Waldlied - Waldhornsklängen - Waldeinsamkeit - Waldwiesen Welt - weite Welt Gottes freie Welt - in der Welt aus der Welt Ferne weite Ferne - blaue Ferne - in der Ferne - aus der weitesten Ferne Mond - Mondlicht - Mondschein - mondhell - das helle Mondlicht
} 
Die Vorliebe für den substantivischen Stil lässt sich generell im Text feststellen, so gelten die Substantive als Inhaltswörter in dem Text. Hier lässt sich z.B. der bewußte Gebrauch von erstarrten phraseologischen Wendungen erwähnen, die sich oft synonym mit Einzelwort ausdrücken lassen, aber der Unterschied besteht in ihrer expressiver Bildhaftigkeit, die mit dem romantischen Stil durchaus korrespondiert (Fleischer, Michel 1977: 77). Die Neigung zum Archaismus, zeitverschobenen Ausdrücken wird übrigens als eines der auffallendsten Merkmale romantischer Sprache bezeichnet. Es entsteht der Eindruck einer Patina der Altertümlichen, als ob die Grenzen der poetischen Illusion überschritten werden könnten. Hier könnte man die erstarrten Redewendungen vorführen, die inhaltlich sehr nah mit den Sprichwörtern stehen und oft zur Gruppe der Wortpaare (Zwillingsformeln) gezählt werden:

...und pfiff dazwischen... dass es einem durch Mark und Bein... Seit diesem Abend hatte ich weder Ruh noch Rast mehr... erhob sich in dem Gebüsch ein rasender Spektakel von Pauken und Trompeten... ...dass es mir durch Leib und Seel ging ...das Herz wollte mir zerspringen von dem Liede vor Scham und vor Schmerz.

Die sprachliche Prägnanz, aber auch ihre Formelhaftigkeit verstärken ihre stilistische Einprägsamkeit. Der Text liefert auch Historismen, welche die altertümelnden Stileffekte im Sinne eines gewissen Zeitkolorits wiedergeben. Hier wäre die Verwendung von Fremdwörtern zu erwähnen, die als Ersetzungen für die deutschen Entsprechungen den stärkeren Stileffekt erzielen:

...ich flickte ein altes Parasol vom Einnehmer... .... ich wollte auch nicht dahinter bleiben in der Galanterie und machte einige artige Kapriolen... Nach Italien, wo die Pomeranzen wachsen. Du vazierst, ... bleib bei uns, da hast du ewige Vakanz.

Der Nominalstil mit den ausgebauten, vor- und nachgestellten Attributen, Appositionen wird im Text bevorzugt, was in Tendenz zu parataktischem Satzbau resultiert:

...das steht ein grosser Herr in Staatskleidern, ein breites Bandelier von Gold und Seide bis an die
Hüften übergehängt, mit einem oben versilberten Stabe in der Hand und einer ausserordentlich lan-
gen gebogenen kurfürstlichen Nase im Gesicht, breit und prachtig wie ein aufgeblasener Puter...
Wir aber rasselten durch die stille Nacht einen Steinweg hinan, ...das einförmige, ewige Gerassel
des Wagens schallte an den Steinwänden weit in die stille Nacht, und die Morgenkühle rieselte mir
durch alle Glieder Der andere Maler trällerte ein Liedchen vor sich hin, und ihre Schleier flatterten
entsetzlich in langen Spitzen wie Nebelstreifen hoch am Himmel empor; das Sausen nahm immer
mehr zu, nur der Springbrunnen vor Eingange plätscherte einsam in einem fort vom Schlosse schal-
te die Tanzmusik nur noch seltener herüber.

Die Wortfolge ist meistens frei, die einzelnen Wortformen lassen sich um die Nominal- und Verbalphrase einordnen. In den Vordergrund der Nominalgruppe treten charakteristische Attributformen. Viele von diesen Worten wären fast als Schlüsselworte aufzufassen. 
Schweifende Morgenstrahlen, Stacheln meiner eignen Gedanken, Ruinen meines Glücks, schimmernde Luft, himmelblaue Winden, das schimmernde Land, unzählige Lerchen, kurfürstliche Nase, prächtige Gegend, mondbeglänzter Platz, mondhelle Nacht, jämmerliches Weinen

Die Appositionen als besondere Attributformen sind im Text zu finden:

ein grosser Herr in Staatskleidern, ein breites Bandelier von Gold und Seide bis an die Hüften überhängt, mit einem oben versilberten Stabe in der Hand und einer ausserordentlich langen gebogenen kurfürstlichen Nase im Gesicht

In der Verbalgruppe erscheinen sehr oft Adverbialbestimmungen, mit oder ohne Präpositionen, beliebt sind in der Erzählung die Partizipialkonstruktionen.

Die Kammerjungfer sah mich kurios an Die kühle Morgenluft weckte mich endlich aus meinen Träumereine. ein dünner Kerl kam wie eingeschossner Ladstock herausgeflogen, als käme diese schöne Frau unten zu mir gegangen oder eigentlich langsam geflogen da kommt auf einmal die Kammerjungfer durch die Dämmerung dahergestrichen. nach mir zurückblickend, durch die Lücke kommt das Tellergeklapper und der Bratenduft in den freudenreichen Schall her herausgezogen.

Das Verb "gehen" kommt oft in Verbindung mit den romantisch empfunden Substantiven vor: Quellen, Bäche, Mühlen, Ströme, Mühlräder, Morgenglocken und Töne "gehen"

Die häufigste Satzfigur bilden aber in der Erzählung Infinitivkonstruktionen.

Das sah ich nun allemal die allerschönste Dame noch heiß und halb verschlafen im schneeweissen Kleide an das offene Fenster hervortreten. Eine Strecke weiter sah ich dann immer die andere Dame am Fenster stehen. sie sieht mich Ärmsten hinter dem Strauche lauschen. wo ich immer unsern Pfarrer so bequem herumgehen sah. Da liessen sich auf einmal die Hörner der zurückkehrenden Jäger von ferne vernehmen, Wie ich eben so philosophiere, höre ich auf einmal unten im gras etwas einherrascheln.

Außer den oben zitierten Möglichkeiten sind bei Einchendorff Infinitive mit "zu" anzutreffen:

Mich zuckte es in allen meinem Gliedern, herunterzuspringen und meine Reputation zu retten. so dass beinah nur die grossen schwarzen Augen zwischen den herabhängenden Locken zu sehen Waren. Anfangs nahm ich mir vor, sobald wir durch den einsamen Wald füren, schnell aus dem Wagen zu springen und zu entlaufen.

In dem zusammengesetzten Satz ist die Relation einer Parataxe verhältnismäßig häufig. Die Sätze sind locker mit Hilfe einer koordinierenden Konjunktion "und", "aber", "oder", "den" aneinander gereiht.

Ich machte ihr ein tiefes Kompliment, und, ich kann nicht anderes sagen, sie dankte mir jedesmal und nickte und blinzelte mit den Augen dazu ganz außerordentlich höflich. Die Leute unten waren ausser sich vor Freude, und ich hielt mich am Ende auch nicht mehr und schrie immer aus Leibeskräften Vivat mit. Ich rannte noch einmal um das ganze Haus herum und rief die Maler, aber nie- 
mand gab Antwort, die Leute aus dem Hause liefen zusammen und gafften mich an, und liesst mich rasch wieder auf den Boden herab, den ich schämte nicht auch, länger wie eine zerbrochene Gabel da über dem Aste zu hängen.

Diese grammatische Konstruktion verursacht, dass sich die Sätze übermäßig ausdehnen.

Von den subordinierend verbundenen Nebensätzen bilden die Attributiv- und Vergleichssätze eine besonders beliebte Satzkonstruktion:

Der Holzweg, auf dem ich fortgelaufen war, hörte auf, Hier war es so einsam, als läge die Welt wohl hundert Meilen weit weg. Auch das Mädchen, das mir den Wein gereicht hatte, ging nur nach dem Dorfe zu, aber sie ging sehr langsam und sah sich zuweilen um, als ob sie war vergessen hätte. Auch einen ganz neuen Hut bekam ich, der funkelte in der Sonne, als wär er mit frischer Butter überschmiert. Es ist, als wäre ich überall eben zu spät gekommen, als hätte die ganze Welt gar nicht auf mich gerechnet.

Dass Eichendorff häufig den Konjunktiv in diesen Sätzen einsetzt, wurden sie zu den irrealen Vergleichsätzen. Der Konjunktiv als Verstärkung des indikativisch Ausgesagten kommt bei Eichendorff auch in anderen konjunktionalen Nebensätzen vor (Köhnke 1975: 287-293). Folgende Sätze sind als irreale Konsekutivsätze einzuordnen:

Wie sie so sprachen, wurde mir so lustig in meinem Sinn, dass ich gleich auch hätte mit studieren mögen. Da kam mir die Welt auf einmal so entsetzlich weit und gross vor und ich so ganz allein darin, dass ich aus Herzensgrunde hätte weinen können. Da fiel mir auf einmal die schöne alte Zeit mit solcher Gewalt aufs Herz, dass ich bitterlich hätte Weinen mögen.

Viel seltener wurden die Konsekutivsätze im Indikativ ausgedrückt:

Dabei sah her mich aber manchmal so kurios mit verdrehten Augen an, dass ich mich ordentlich vor Ihm zu fürchten anfing und ihn plötzlich wieder losliess.

... und zuletzt geriet ich in ein solches entsetzliches und unaufhaltsames Schlafen, dass gar kein Rat mehr war.

Kausal- Konzessivsätze scheinen bei Eichendorff die Relationen auszudrücken, die vielmehr stärker in Wirklichkeit verankert sind:

Da ich keinen Menschen erblickte, stieg ich über den niedrigen Gartenzaun was mir gar wohl bekam, da ich seit einigen Tagen schon nicht mehr vernünftig gespeist hatte, und die Magd sich bekreuzte, weil es aussah wie ein Gespenst, das auf einem dreibeinigen Pferde reitet. Wo ich hintrat, hatten die Leute eine grosse Ehrbietung vor mir, obgleich schon alle wussten, dass ich keinen Heller in der Tasche hatte.

Objektsätze im Konjunktiv veranschaulichen das Außergewöhnliche:

Ich konnte mich gar nicht besinnen, wo ich eigentlich wäre. wenn er immer zu sagen pflegte, da? in Italien einen Rosinen von selbst in den Mund wüchsen. Ich schrie zum Wagen heraus auf den Postillon: wohin er den fahre? Ich war ganz verblüfft und sagte endlich, dass ich soeben auf einer Reise 
nach Italien begriffen wäre. da wurde mir auf einmal ganz grauslich, ich merkte wohl, dass er verrückt war. Ich aber merkte nun, dass es Prager Studenten waren

Temporalsätze sind meistens Indikativ bezogen:

Als wir an das Ufer kamen, war schon alles zur Abfahrt bereit. Ich aber jauchzte laut auf, als ich auf einmal wieder die Donau so recht vor mir sah. Ich blickte, während das Schiff so fortflog und die Wellen unter mir rauscht en und schäumten Als er mich erblickte, kam er mit ausgebreiteten Armen auf mich los. Sobald sie mich erblickte, fing sie an, tiefe Knickse zu machen...

Typisch für den Satzbau im Text sind umfangreiche Satzgebilde mit reich ausgebauten Innenteilen, Gebrauch von Nebensätzen verschieden Grades, den sogenannten Treppensätzen innerhalb eines Schachtelsatzes, wie z.B.:

Sooft der Gärtner fort und ich allein war, zog ich sogleich mein kurzes Tabakspfeifchen heraus, setzte mich hin und sann auf schöne höfliche Redensarten, wie ich die eine junge, schöne Dame, die mich in das Schloss mitbrachte, unterhalten wollte, wenn ich ein Kavalier wäre und mit ihr hier herumginge.

Kennzeichnend für die Sprache Eichendorffs ist vor allem seine Vorliebe für gewählte, wiederkehrende Substantive, häufig Substantivkomposita, die als ethischnormative Begriffe des romantischen dichterischen Sprachstils aufgefasst werden könnten. Neben den romantischen Schlüsselworten findet man aber bei ihm auch eine Fülle von Attributgruppen, die auf seinen individuellen Wortschatz und Wortzusammenstellung verweisen. Im Bereich des Satzes treten vor allem die reich ausgebauten längeren Perioden in den Vordergrund; ihre Gliedsätze, oft im Konjunktiv werden meistens durch subordinierende Konjunktionen eingeleitet.

In der Darstellungsart der großen Reise werden die Symphatien des katholisch gesinnten Dichters sichtbar. Seine Sehnsucht nach der mittelalterlichen Vergangenheit ließ seinen Helden die Reise von Wien nach Rom unternehmen, indem retrospektiv die alte Karte durchgefahren wurde. Der Gattungstitel Novelle darf uns nicht zum Vorgriff auf die Novelle des Realismus verleiten, die Probleme und Konflikte zeitrealistisch exponiert. Unzweifelhaft liest sich manches eher wie ein Märchen, es gibt nicht psychologisch motivierte, sondern märchenhaft glückliche Fügungen, die das Schicksal des Taugenichts gestalten und Landschaften, die in ihrer märchenhaften Abstraktheit - Schlösser, Gärten - austauschbar erscheinen.

Solche Bemerkungen zur Form führen uns zur sprachlichen Verfasstheit des Textes. Die literaturwissenschaftliche Forschung hat ja immer unterstrichen, dass die seelische Verfassung des Taugenichts Spiegelbild auch des momentan herrschenden Naturzustandes und der von ihm beschriebenen Landschaftseindrücke sei. Auch auf den Antirealismus, auf den lyrischen Blick nach innen hat sie Wert gelegt, wenn sie, wie z.B. Katharina Tiemeyer unterstreicht, dass der von Wien nach Italien reisende Taugenichts kein Italienfahrer der klassischen Generation sei, der sich mit Andacht und Studium der Antike widmete. Er schläft, genauer er träumt beinahe die ganze Zeit in seiner Kutsche. 


\section{Literatur}

Admoni, W. (1982): Der deutsche Satzbau. München: Beck.

Boost, K. (1949): Der deutsche Satz. Die Satzverflechtung. In: Deutschunterricht. 3, S. 7-15.

Bormann, A. von (1988): Aus dem Leben eines Taugenichts. In: Erzählungen und Novellen des 19 Jahrhunderts. Bd.1. Stuttgart: Reclam.

Eroms, H.-W. (2000): Syntax der deutschen Sprache. Berlin: de Gruyter.

Eroms, H.-W. (2008): Stil und Stilistik. Eine Einführung. Berlin: E. Schmidt.

Fleischer, W., Michel, G. (1977): Stilistik der deutschen Gegenwartssprache. Leipzig: VEB Bibliographisches Institut.

Köhnke, K. (1975): Zum Gebrauch des Konjunktivs in Eichendorffs Erzählungen. In: Akten des V Internationalen Germanisten-Kongresses Cambridge. Heft 2, S. 287-293.

Maingueneau, D. (2000): Linguistische Grundbegriffe zur Analyse literarischer Werke. Tübingen: Narr. Riesel, E., Schendels, E, (1970): Deutsche Stilistik. Moskau: Verlag Hochschule.

Silman, T. (1974): Probleme der Textlinguistik: Einführung und exemplarische Analyse. Heidelberg: Quelle \& Meyer.

Stöcklein, P. (1987): Joseph von Eichendorff: mit Selbstzeugnissen und Bilddokumenten. Reinbek bei Hamburg: Rowohlt. 